\title{
ASSESSING THE TRANSFER OF SIMULATOR TRAINED SKILLS TO REAL VEHICLE CONTROL
}

\author{
Barry Coutermarsh, Kelley MacDonald, \& Sally Shoop \\ U.S. Army Engineer Research and Development Center, Cold Regions Research and \\ Engineering Laboratory (ERDC/CRREL) \\ Hanover, New Hampshire, USA \\ Email: Barry.A.Coutermarsh@usace.army.mil
}

\begin{abstract}
Summary: The Army Cold Regions Research and Engineering Laboratory is conducting a project to investigate the value of a motion based simulator in teaching vehicle control for off-road driving conditions. A primary goal is to reduce accidents caused by the loss of control of high center-of-gravity military vehicles in situations normally not found in the civilian driving experience. This study presents data from a two year portion of our work to develop metrics to assess the effectiveness of simulator training for developing vehicle control skills. For the first year, 10 drivers were trained using a simulator in an accident avoidance (AA) maneuver. Their performance was compared against 10 untrained drivers in a real vehicle. The second year 5 trained drivers from the first study were given sparse sustainment training in the simulator and again compared against 5 untrained drivers in a real vehicle. We considered metrics specifically related to the vehicle control aspects of the training to determine if the trainee acquired the necessary muscle memory to correctly implement the various vehicle control steps involved in the maneuver. We also briefly describe the participant's views on their training experience.
\end{abstract}

\section{OBJECTIVE}

A common driving condition for military drivers is either off-road or on unpaved poorly designed and maintained roads. The vehicles are normally non-ABS equipped and frequently heavily loaded with a high center-of-gravity making them inherently at risk of rolling over. The drivers are frequently operating under high-stress conditions with unexpected external factors impacting their driving conditions. Maintaining vehicle control under these conditions is a primary concern.

We have partnered with the Team O’Neil Rally School and Car Control Center to develop vehicle control training procedures and techniques common to high-speed off-road rally car drivers. We are investigating car control techniques that could be useful in controlling military vehicles in off-road applications and that can be implemented in an automated simulation training environment.

The work described here spans a two year period where students were trained using an Accident Avoidance (AA) scenario in a simulator with emphasis on the steps necessary for vehicle control throughout the maneuver. The goal was to use the simulator to develop muscle memory in the students so the necessary driver inputs would become automatic during the real vehicle tests. Their performance was then compared against untrained drivers executing the AA maneuver in a 
real vehicle. We developed a set of objective assessment metrics specific to the vehicle control steps trained that we hoped would tell us if the simulator training was effective. The work here presents the metrics measured and shows the trends between the trained and untrained drivers using those metrics.

\section{METHODS}

The first year 10 students were trained by an instructor using the SimCraft 3-axis motion base simulator (Figure 1) running CarSim DS software. The students attended a 30 minute class every week for a total of 10 weeks. The training focused on vehicle control and various weight transfer techniques as well as practice in the AA maneuver itself. These 10 trained drivers were compared against 10 untrained drivers in a real vehicle running the same AA scenario. The second year of testing was sustainment training. The goal was to determine if short infrequent training sessions could sustain the necessary skills to perform the AA maneuver correctly. 5 trained students from the first year's testing were given an approximately 10 minute long refresher session in the simulator, with an instructor, once every three or four weeks over a 7 month period. These five students were then compared against five untrained drivers in real vehicle tests. In both cases the students were people working at our research laboratory with ages from the mid 20's to lower 60’s. All had driven for most of their adult life.
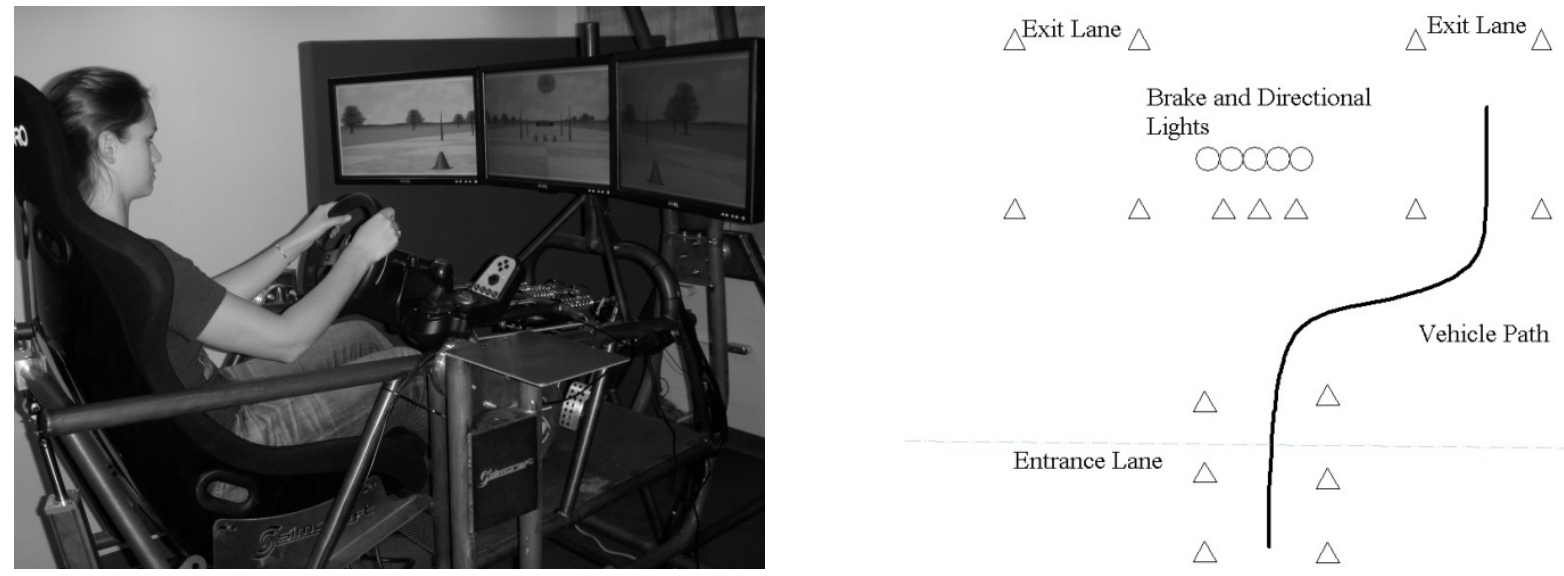

Figure 1. The SimCraft motion simulator (left) and the AA maneuver layout (right)

The AA maneuver consisted of driving a front wheel drive, non ABS equipped vehicle at a target speed between 30 and $35 \mathrm{mph}$, depending upon the conditions, between a central lane defined by traffic cones which ended in three central cones blocking the lane. There were two exit lanes to the left and right of the central cones, one of which the driver maneuvers into based upon light signals placed behind the central cones. As the vehicle approaches down the central lane red lights appear indicating the driver should begin braking. The red lights go off and simultaneously a green light on either the right or left comes on indicating which lane the driver should exit to. This configuration was used in both the simulator and real vehicle tests and is shown in Figure 1. The vehicle control steps that were taught are shown in Table 1. 
Table 1. External events and driver responses

\begin{tabular}{|c|c|}
\hline External Condition & Driver Task \\
\hline Approach down entrance lane & Maintain target speed, foot on gas pedal \\
\hline Red lights on & Off gas pedal and apply brake, eyes on lights \\
\hline Red lights off and green light on & $\begin{array}{l}\text { Release brake, turn to appropriate lane, look to } \\
\text { center of entrance to exit lane }\end{array}$ \\
\hline Vehicle at entrance to exit lane & Turn back into exit lane, look down exit lane \\
\hline Vehicle in and aligned with exit lane & $\begin{array}{l}\text { Apply gas, look far down lane where vehicle } \\
\text { should go }\end{array}$ \\
\hline
\end{tabular}

Various driver inputs were recorded during both the simulated and real vehicle testing. They consisted of steering wheel angle, brake and gas pedal response. These were measured along with vehicle speed. The simulator data also had the time the various light signals were activated but this was not available to us for the first year of real vehicle testing. During the second year of testing we did have this capability.

The difficulty of the maneuver is greatly affected by the vehicle speed, the timing of the lights and the ground surface conditions. The timing of brake, gas and steering are the critical driver inputs in performing the AA maneuver correctly. Eye gaze is also an important factor and although it was stressed in the training it was one we couldn't measure well. The reaction time of the driver to the light inputs is a contributing factor that will affect the difficulty of the maneuver.

The sequence of driver inputs stressed in this training were to apply the brakes as hard and as quickly as possible when the braking lights appeared and release them when the turn light appears since with non-ABS brakes it is impossible to steer with the front tires locked and sliding. Then look and steer to the exit lane, steer back to drive down the lane while looking far down the lane after which apply some gas to stabilize the rear of the vehicle. The steps are taught in a specific sequence and are designed to dynamically shift the vehicle weight first to the front axle to assist in lateral force for turning and then to the rear axle at the last turn to assist in preventing the rear of the vehicle from sliding.

\section{ANALYSIS AND METRICS}

Based upon the critical steps the driver should perform during the AA maneuver, the metrics we thought were important to look at were associated with the timing of the brake, steering and throttle inputs. They are listed in Table 2 below. We looked at the peak brake force and the slope of that force when applying and releasing the brake pedal as a measure of the confidence the driver exhibited during braking. 
Table 2. Desired driver task and assessment metric

\begin{tabular}{ll}
\hline Desired Driver Task & Metric \\
\hline Constant target approach speed & Vehicle speed at braking \\
Steer only after brake release & Time between brake release and steer \\
Quick and hard brake application at light & Slope to peak brake force \\
Quick release of brake at turn light & Peak brake force \\
Overall short time on brakes & Slope from peak brake to release \\
\hline
\end{tabular}

Figure 2 shows vehicle speed just before braking was initiated and the time between brake release and steering. There is a consistent but small trend between the two years of slightly higher speed with the trained drivers. Both groups were approximately four to five mph faster the first year.
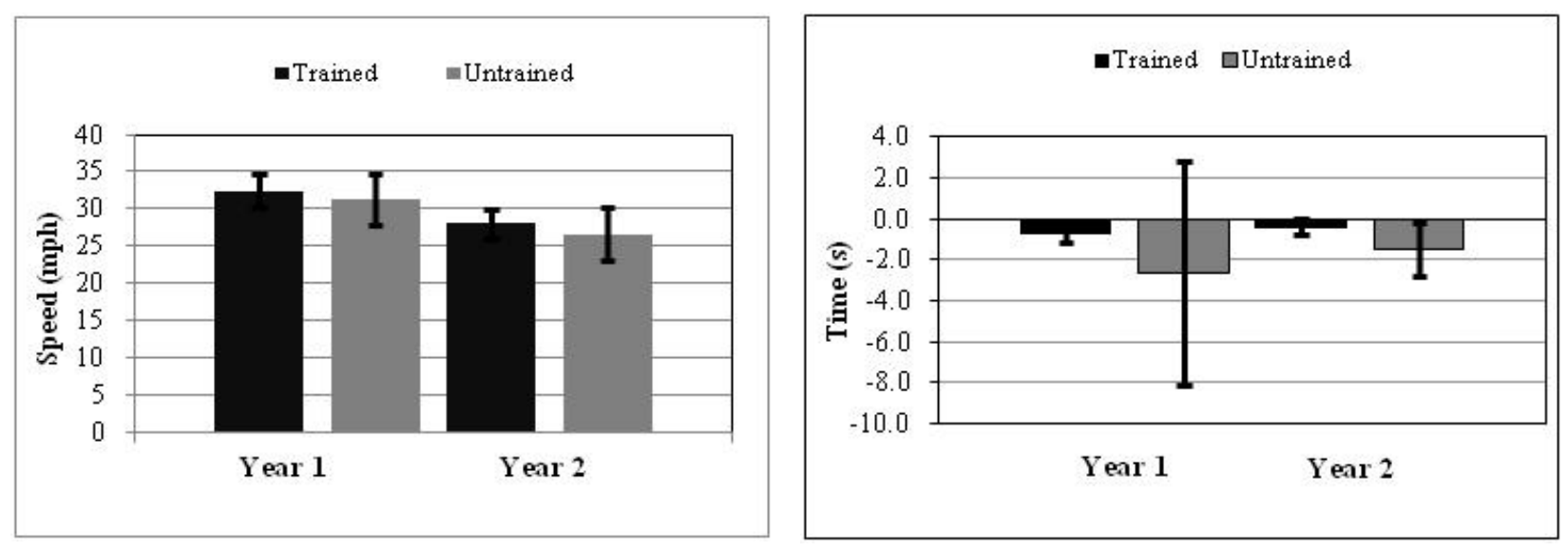

Figure 2. Speed at brake light (left) and time time between brake release and steering (right)

The right side of Figure 2 is the time between brake release and steering. A negative time here indicates that steering began before the brake was fully released. It can be seen that both groups in both years exhibit this characteristic. The ideal time would be zero for this input which would indicate that the driver started steering immediately after releasing the brakes. The trained drivers were substantially better at this input with times closer to zero and lower standard deviations than the untrained. Another training goal in this maneuver was to quickly and forcefully apply the brakes during the braking segment to achieve the maximum speed reduction. Equally important was to quickly release them as the turn light came on to allow a quick transition to steering without the front tires locked up. Figure 3 shows the slopes from no braking to peak braking force and from peak braking force to release. These indicate the brake application and release speeds and a small slope value generally indicates a slow response. These charts show a trend of faster mean responses for the trained drivers however the standard deviations are generally relatively high. 

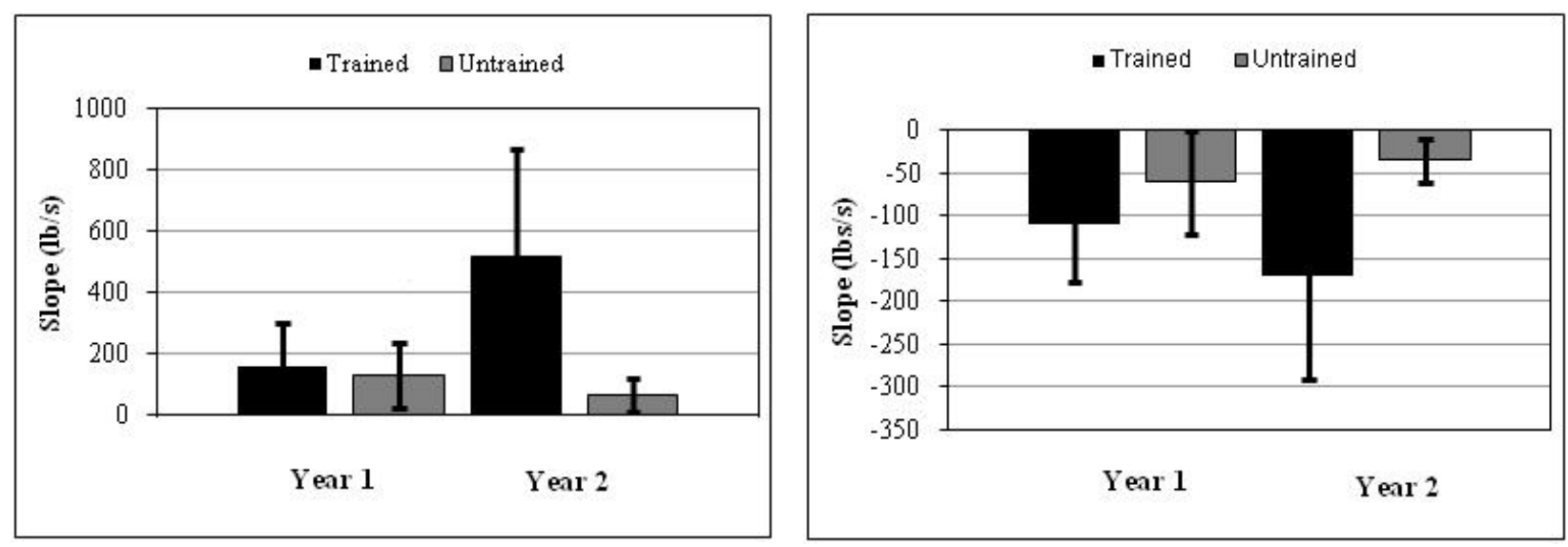

Figure 3. Slope from no braking to peak braking (left) and from peak brake to release (right)
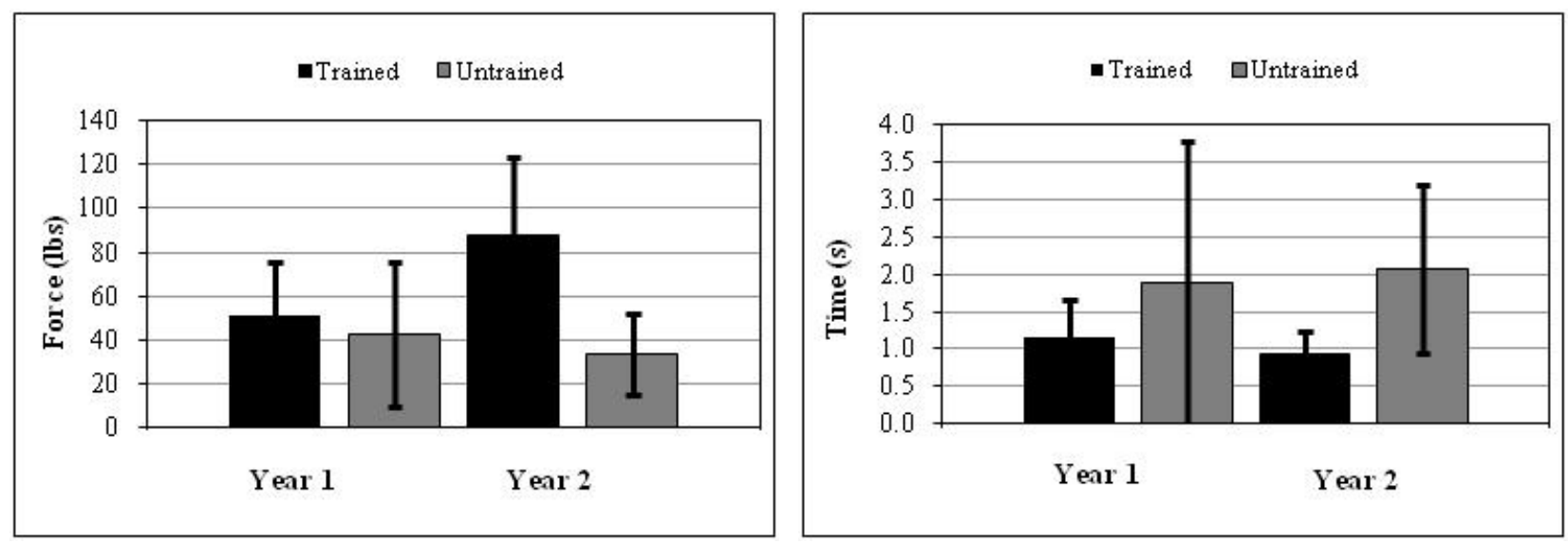

Figure 4. Peak brake force (left) and brake duration (right)

Figure 4 is the peak brake force and total braking time or brake duration. The figure shows that trained drivers exhibited a higher mean force when braking with shorter mean brake duration in both years but again with high standard deviations. These values were greatly affected by a few instances where the brake pedal was never fully released until late in the maneuver.

Throughout the data there were a few distributions that had smilar shapes but were skewed from the normal. We performed a Mann-Whitney U two-tailed test on each of the metrics to assess the null hypothesis that there was no difference between the trained and untrained driver groups. The results are presented in Table 3 and show a significant difference between the groups for each metric at the $95 \%$ confidence level. 
Table 3. Results of a Mann-Whitney W test on the chosen metrics

\begin{tabular}{lcccc}
\hline \multirow{2}{*}{\multicolumn{1}{c}{ Metric }} & \multicolumn{4}{c}{ Statistics } \\
\cline { 2 - 5 } & $\begin{array}{c}\text { Median } \\
\text { Trained }\end{array}$ & $\begin{array}{c}\text { Median } \\
\text { Untrained }\end{array}$ & U & P \\
\hline Speed at brake light & 31.37 & 28.53 & 854 & .0214 \\
Time between brake release and steer & -0.554 & -1.433 & 560 & $<0.0001$ \\
Slope from no brake to peak brake & 185.3 & 68.71 & 659 & 0.0002 \\
Slope from peak brake to release & -98.41 & -34.31 & 443 & $<0.0001$ \\
Peak brake force & 56.85 & 33.42 & 635 & 0.0001 \\
Brake duration & 0.9770 & 1.536 & 668.5 & 0.0003 \\
\hline
\end{tabular}

\section{CONCLUSIONS AND DISCUSSION}

The initial work described above is a proof-of-concept look at training with a simulator, assessing that training and learning some of the problems associated with such work. Our AA maneuver was meant to simulate a situation where a driver encounters an unexpected obstacle and must quickly brake and turn to avoid hitting it. In our tests we tried to design it to place enough stress on the driver so it would be a measure of muscle memory. The difficulty of the test is greatly affected by the speed of the vehicle and the timing of the lights relative to the surface conditions. If the speed is too slow or the light timing is off then the maneuver can be either impossible to perform or much too easy requiring no automatic response. We found a variation of 3 to $5 \mathrm{mph}$ to be significant. Our timing was set by expert instructors based upon the prevailing conditions but it was ultimately determined by what felt right to them for the conditions at the time of the tests.

The data presented here are related to the vehicle control tasks that were emphasized in the driver training. The trends presented seem to indicate that the trained drivers exhibited a better execution of the tasks than ones with no training. The question still remains as to if learning these tasks would make a driver better at controlling a vehicle in a different situation. We interviewed the trained participants in the study and all but one felt the training had influenced the way they drive. These results were similar to those found in Lindsey and Barron (2005).

In this study we used a motion based simulator but feel that for the AA maneuver motion is unnecessary and the participants agreed. They also felt the instructor was an integral part of the instruction but practicing the maneuver in the simulator was judged important. We find it difficult to obtain literature on motion based vehicle training for vehicle control applications. Most past work seems to be in situational awareness training, especially in military applications.

In their work, Bowen, et al (Bowen, et al (2006)) point out that past work has divided skill-based driving tasks into two categories; "tracking” where visual cues are sufficient and motion is unnecessary and "disturbance" where motion is important. The AA maneuver would fall into the tracking category. Our future work will fall into the disturbance category where we feel the vehicle motion, e.g. imminent roll-over, will impact and initiate the driver's response. In the aviation community, McCauley's review of past work (McCauley, 2006) concludes that in helicopter pilot training motion improves performance in the simulator but there is little scientific evidence that supports its training effectiveness. 
The future direction of our work in this project will develop an automated simulator based teaching program for vehicle control. The program will train a driver in various scenarios designed to teach progressive vehicle control skills such as soft shoulder departures, side slope control and brake-and-avoid maneuvers with both ABS and non-ABS braking. The vehicles will range from a civilian type sedan to military HMMWV's with and without a gunner's turret.

\section{REFERENCES}

Bowen, Shane A., Brian P. Oakley and John S. Barnett (2006). Effects of Motion on Skill Acquisition in Future Simulators. Study Report 2006-07, United States Army Research Institute for the Behavioral Sciences, Arlington, VA.

Lindsey, Jeffrey T. and Ann E. Barron (2005). The Perceptions of Emergency Vehicle Drivers Using Simulation in Driver Training. Proceedings of the Third International Driving Symposium on Human Factors in Driver Assessment, Training and Vehicle Design, Rockport, Maine, 66-72.

McCauley, Michael E. (2006). Do Army Helicopter Training Simulators Need Motion Bases? Technical Report 1176, United States Army Research Institute for the Behavioral and Social Sciences, Arlington, VA. 